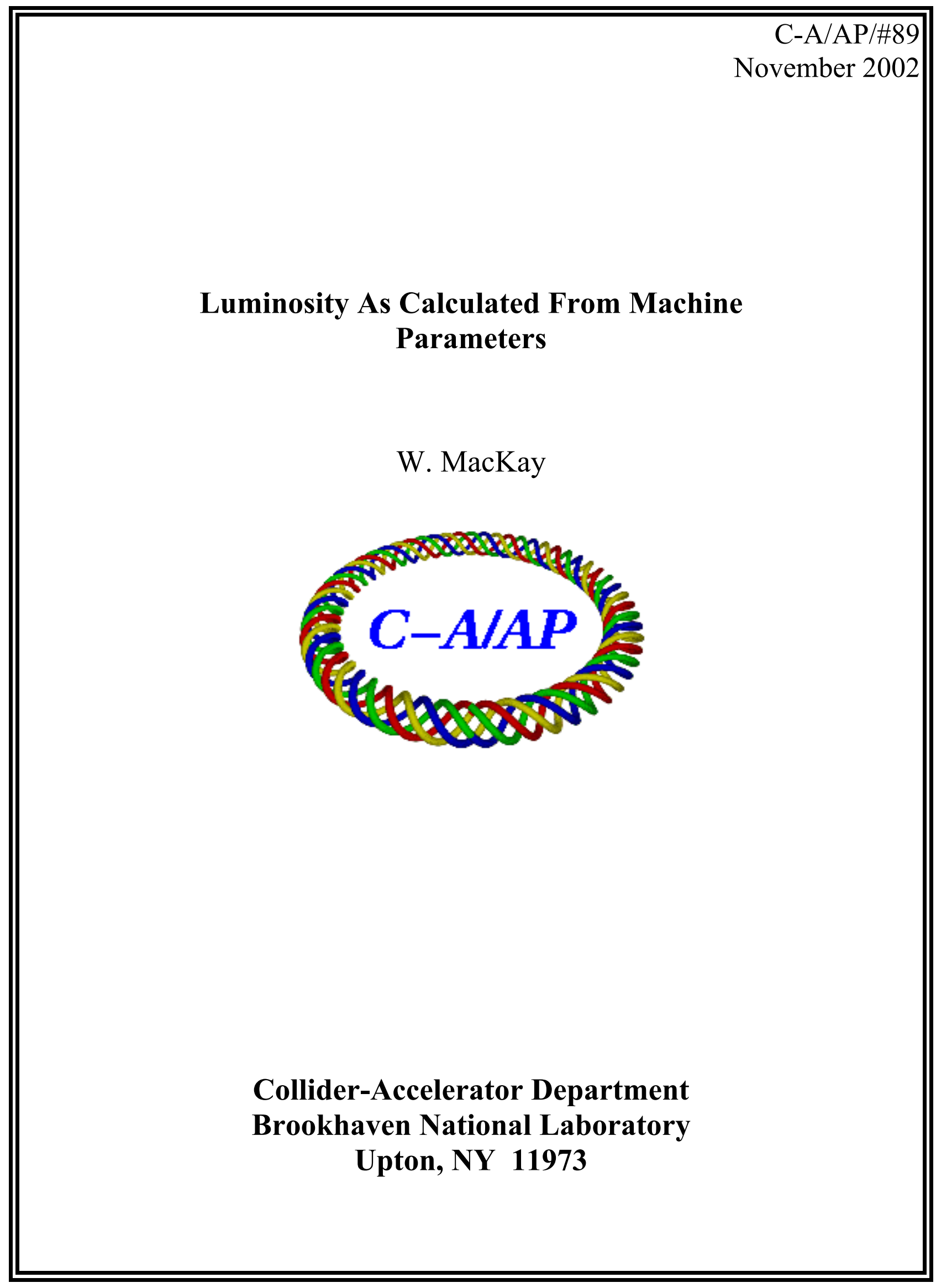


14 Dec., 1999

\title{
LUMINOSITY AS CALCULATED FROM MACHINE PARAMETERS
}

\author{
W. W. MACKAY
}

\section{INTRODUCTION}

The luminosity rate of interactions, at time $t$, between two colliding beams may be easily understood by treating one beam as a target, with number density $\rho_{2}\left(\overrightarrow{\mathbf{X}}_{2}, t\right)$, and the other as an incident beam, whose flux density is $v \rho_{1}\left(\overrightarrow{\mathbf{X}}_{1}, t\right)$, where

$$
v=\left|\vec{v}_{1}-\vec{v}_{2}\right|
$$

is the relative velocity. ${ }^{1}$ The coordinates $\mathbf{X}_{2}$ and $\mathbf{X}_{1}$ are points in the six dimensional phase space $\left(x, x^{\prime}, y, y^{\prime}, s, \delta\right)$. The interaction rate may then be written as

$$
\frac{d N}{d t}=\int \sigma\left(\overrightarrow{\mathbf{X}}_{2}, \overrightarrow{\mathbf{X}}_{1}\right) \rho_{2}\left(\overrightarrow{\mathbf{X}}_{2}, t\right)\left|\vec{v}_{1}-\vec{v}_{2}\right| \rho_{1}\left(\overrightarrow{\mathbf{X}}_{1}, t\right) d^{6} \mathbf{X}_{2} d^{6} \mathbf{X}_{1},
$$

where $\sigma$ is the cross section for single particle interactions.

Of course, for the actual rate seen by an experiment one must fold in the detector acceptance and efficiency for a desired reaction by integrating the differential cross section times acceptance and efficiency over the the phase space of final particles.

For most high energy collisions the probability of interaction is extremely small, unless the particles are very close, e. g.,

$$
\left|\vec{x}_{1}-\vec{x}_{2}\right| \lesssim 10^{-15} \mathrm{~m} \text {. }
$$

This is much less than the size of a typical beam $\left(\gtrsim 10^{-6} \mathrm{~m}\right)$. It is therefore reasonable to approximate the cross section by

$$
\sigma\left(\overrightarrow{\mathbf{X}}_{2}, \overrightarrow{\mathbf{X}}_{1}\right) \simeq \sigma\left(\vec{p}_{2}, \vec{p}_{1}\right) \delta^{3}\left(\vec{x}_{1}-\vec{x}_{2}\right) .
$$

If the beams are almost monoenergetic, $\sigma$ may be factored outside the integral and replaced by the total cross section, so that

$$
\frac{d N}{d t} \simeq\left|\vec{v}_{1}-\vec{v}_{2}\right| \sigma\left(\vec{p}_{2}, \vec{p}_{1}\right) \int \rho_{2}(\vec{x}, t) \rho_{1}(\vec{x}, t) d^{3} x .
$$

If the bunch shape does not change appreciably while the opposing bunches overlap, then the time dependence of the $i^{\text {th }}$ densities may be written as

$$
\rho_{i}(\vec{x}, t)=\rho_{i}\left(\vec{x}-\vec{v}_{i} t\right)
$$

\footnotetext{
${ }^{1}$ For relativistic colliding beams this relative velocity is the difference of the two velocities $v \simeq$ $|c-(-c)|=2 c$ as calculated in the between the two beams. Perhaps the best way to think of this is that an observer in the rest system of the collision point sees the bunches pass through each other at twice the effective speed as a single bunch.
} 
and the total number of interactions from one bunch crossing is

$$
N=\left|\vec{v}_{1}-\vec{v}_{2}\right| \sigma \int \rho_{2}\left(\vec{x}-\vec{v}_{2} t\right) \rho_{1}\left(\vec{x}-\vec{v}_{1} t\right) d^{3} x d t
$$

When there are $N_{b}$ equally spaced bunches per beam in a circular collider whose revolution frequency is $f_{0}$, the interaction rate per interaction region is

$$
\frac{d N}{d t}=\left|\vec{v}_{1}-\vec{v}_{2}\right| f_{0} N_{b} \sigma \int \rho_{2}\left(\vec{x}-\vec{v}_{2} t\right) \rho_{1}\left(\vec{x}-\vec{v}_{1} t\right) d^{3} x d t
$$

The ratio of interaction rate to total cross section is called the instantaneous luminosity

$$
\mathcal{L}=\left|\vec{v}_{1}-\vec{v}_{2}\right| f_{0} N_{b} \int \rho_{2}\left(\vec{x}-\vec{v}_{2} t\right) \rho_{1}\left(\vec{x}-\vec{v}_{1} t\right) d^{3} x d t
$$

which for high energy collisions in the center of mass system becomes

$$
\mathcal{L}=2 c f_{0} N_{b} \int \rho_{2}(x, y, z-c t) \rho_{1}(x, y, z+c t) d^{3} x d t,
$$

where beam- 2 moves in the $+z$ direction. The total or integrated luminosity refers to the instantaneous luminosity integrated over the time of the experiment.

If the bunch densities change shape in the overlap region, then the simplification of Eq. 6 is invalid and the luminosity must be calculated from

$$
\mathcal{L}=2 c f_{0} N_{b} \int \rho_{2}(x, y, z, t) \rho_{1}(x, y, z, t) d^{3} x d t .
$$

If the beams cross where the transverse beta-functions both have minima, then the minima of the beta-functions should not be smaller than the bunch length, otherwise too much of the overlap region has a lower density, and the peak luminosity is degraded. The beta-function about an interaction point goes like

$$
\beta(s)=\beta^{\star}+\frac{s^{2}}{\beta^{*}},
$$

with the minimum value of $\beta^{*}$ at the interaction point $(s=0)$. For bunches of length $l_{b}$, the overlap occurs between $s= \pm l_{b} / 2$. If $l_{b}=\beta^{\star}$ the longitudinal centers of the bunches cross at $s=0$ where $\beta=\beta^{\star}$ and the opposite ends of the bunches cross where $\beta=1.25 \beta^{\star}$.

\section{Gaussian BeAm Distributions}

For one transverse horizontal degree of freedom the Courant-Snyder invariant is

$$
\begin{aligned}
W_{x} & =\left(\begin{array}{ll}
x_{\beta} & x_{\beta}^{\prime}
\end{array}\right)\left(\begin{array}{cc}
\gamma_{x} & \alpha_{x} \\
\alpha_{x} & \beta_{x}
\end{array}\right)\left(\begin{array}{l}
x_{\beta} \\
x_{\beta}^{\prime}
\end{array}\right) \\
& =\gamma x_{\beta}^{2}+2 \alpha x_{\beta} x_{\beta}^{\prime}+\beta x^{\prime 2},
\end{aligned}
$$

where $\beta_{x}, \alpha_{x}$, and $\gamma_{x}=\left(1+\alpha_{x}^{2}\right) / \beta_{x}$ are the Twiss parameters for betatron motion, and $x_{\beta}$ and $x_{\beta}^{\prime}$ are the transverse betatron coordinate and angle in a paraxial phase 
space. A Gaussian distribution of particles undergoing betatron oscillations for this degree of freedom may be written as

$$
f_{\beta}\left(x_{\beta}, x_{\beta}^{\prime}\right)=\frac{N_{z}}{2 \pi \epsilon} e^{-\frac{1}{2} W_{x} / \epsilon_{x}},
$$

where $N$ is the number of particles and $\pi \epsilon_{x}$ is the rms horizontal emittance.

Similarly for the longitudinal motion we may write an invariant

$$
\begin{aligned}
W_{z} & =\left(\begin{array}{ll}
z & \delta
\end{array}\right)\left(\begin{array}{cc}
\gamma_{z} & \alpha_{z} \\
\alpha_{z} & \beta_{z}
\end{array}\right)\left(\begin{array}{l}
z \\
\delta
\end{array}\right) \\
& =\gamma_{z} z^{2}+2 \alpha_{z} z \delta+\beta_{z} \delta^{2}
\end{aligned}
$$

The corresponding longitudinal distribution function for a Gaussian bunch is

$$
f_{z}(z, \delta)=\frac{N}{2 \pi \epsilon_{z}} e^{-\frac{1}{2} W_{z} / \epsilon_{z}},
$$

where $z=s-v t$ and $\delta=\Delta p / p$ are respectively the longitudinal and fractional momentum deviations of a particle from the design particle when it passes the coordinate $s$.

The total transverse coordinates are the sum of the betatron coordinates and the effect of dispersion:

$$
X=\left(\begin{array}{c}
x \\
x^{\prime}
\end{array}\right)=\left(\begin{array}{c}
x_{\beta} \\
x_{\beta}^{\prime}
\end{array}\right)+\left(\begin{array}{ll}
0 & \eta_{x} \\
0 & \eta_{x}^{\prime}
\end{array}\right)\left(\begin{array}{l}
z \\
\delta
\end{array}\right),
$$

where $\eta_{x}$ and $\eta_{x}^{\prime}$ are the horizontal closed-orbit dispersion functions. Since the Jacobian of the transformation from coordinates $\left(x_{\beta}, x_{\beta}^{\prime}, z, \delta\right)$ to $\left(x, x^{\prime}, z, \delta\right)$ is one, we may write a combined distribution function as

$$
f\left(x, x^{\prime}, z, \delta\right)=\frac{N}{(2 \pi)^{2} \epsilon_{x} \epsilon_{z}} e^{-\frac{1}{2}\left[(X-D Z)^{\mathrm{T}} \Xi_{\beta}(X-D Z)+Z^{\mathrm{T}} \Xi_{z} Z\right]}
$$

with the definitions:

$$
\begin{aligned}
& X=\left(\begin{array}{c}
x \\
x^{\prime}
\end{array}\right), \quad Z=\left(\begin{array}{l}
z \\
\delta
\end{array}\right), \quad D=\left(\begin{array}{cc}
0 & \eta_{x} \\
0 & \eta_{x}^{\prime}
\end{array}\right), \\
& \Xi_{\beta}=\frac{1}{\epsilon_{x}}\left(\begin{array}{cc}
\gamma_{x} & \alpha_{x} \\
\alpha_{x} & \beta_{x}
\end{array}\right), \quad \text { and } \quad \Xi_{z}=\frac{1}{\epsilon_{z}}\left(\begin{array}{cc}
\gamma_{z} & \alpha_{z} \\
\alpha_{z} & \beta_{z}
\end{array}\right) .
\end{aligned}
$$

Rearranging terms in the distribution and adding in a similar distribution function for uncoupled vertical betatron motion yields the equation

$$
f\left(x, x^{\prime}, y, y^{\prime}, z, \delta\right)=\frac{N}{(2 \pi)^{3} \epsilon_{x} \epsilon_{y} \epsilon_{z}} e^{-\frac{1}{2} \hat{\mathrm{X}}^{\mathrm{T}} \Xi \hat{\mathrm{X}}}
$$


where

$$
\begin{aligned}
\hat{\mathrm{X}}=\left(\begin{array}{c}
x \\
x^{\prime} \\
y \\
y^{\prime} \\
z \\
\delta
\end{array}\right), \text { and } \\
\Xi=\left(\begin{array}{ccccccc}
\frac{\gamma_{x}}{\epsilon_{x}} & \frac{\alpha_{x}}{\epsilon_{x}} & 0 & 0 & 0 & -\frac{\gamma_{x} \eta_{x}+\alpha_{x} \eta_{x}^{\prime}}{\epsilon_{x}} \\
\frac{\alpha_{x}}{\epsilon_{x}} & \frac{\beta_{x}}{\epsilon_{x}} & 0 & 0 & 0 & -\frac{\alpha_{x} \eta_{x}+\beta_{x} \eta_{x}^{\prime}}{\epsilon_{x}} \\
0 & 0 & \frac{\gamma_{y}}{\epsilon_{y}} & \frac{\alpha_{y}}{\epsilon_{y}} & 0 & 0 \\
0 & 0 & \frac{\alpha_{y}}{\epsilon_{y}} & \frac{\beta_{y}}{\epsilon_{y}} & 0 & 0 \\
0 & 0 & 0 & 0 & \frac{\gamma_{z}}{\epsilon_{z}} & \frac{\alpha_{z}}{\epsilon_{z}} \\
-\frac{\gamma_{x} \eta_{x}+\alpha_{x} \eta_{x}^{\prime}}{\epsilon_{x}} & -\frac{\alpha_{x} \eta_{x}+\beta_{x} \eta_{x}^{\prime}}{\epsilon_{x}} & 0 & 0 & \frac{\alpha_{z}}{\epsilon_{z}} & \frac{\beta_{z}}{\epsilon_{z}}+\frac{H}{\epsilon_{x}}
\end{array}\right),
\end{aligned}
$$

with the function $H=\gamma_{x} \eta_{x}^{2}+2 \alpha_{x} \eta_{x} \eta_{x}^{\prime}+\beta_{x} \eta_{x}^{\prime 2}$. The function $H$ is sometimes called the dispersion invariant since it is constant through regions of the lattice with no bends (i. e., straight sections). With a bit of algebra one can show that

$$
\operatorname{det}(\Xi)=\frac{1}{\epsilon_{x}^{2} \epsilon_{y}^{2} \epsilon_{z}^{2}} .
$$

The beam sigma matrix $(\Sigma)$ or variance matrix for the distribution is just the inverse of $\Xi$ :

$$
\Sigma=\left(\begin{array}{cccccc}
\beta_{x} \epsilon_{x}+\eta_{x}^{2} \sigma_{\delta}^{2} & -\alpha_{x} \epsilon_{x}+\eta_{x} \eta_{x}^{\prime} \sigma_{\delta}^{2} & 0 & 0 & -\eta_{x} \alpha_{z} \epsilon_{z} & \eta_{x} \sigma_{\delta}^{2} \\
-\alpha_{x} \epsilon_{x}+\eta_{x} \eta_{x}^{\prime} \sigma_{\delta}^{2} & \gamma_{x} \epsilon_{x}+\eta_{x}^{\prime 2} \sigma_{\delta}^{2} & 0 & 0 & -\eta_{x}^{\prime} \alpha_{z} \epsilon_{z} & \eta_{x}^{\prime} \sigma_{\delta}^{2} \\
0 & 0 & \beta_{y} \epsilon_{y} & -\alpha_{y} \epsilon_{y} & 0 & 0 \\
0 & 0 & -\alpha_{y} \epsilon_{y} & \gamma_{y} \epsilon_{y} & 0 & 0 \\
-\eta_{x} \alpha_{z} \epsilon_{z} & -\eta_{x}^{\prime} \alpha_{z} \epsilon_{z} & 0 & 0 & \beta_{z} \epsilon_{z} & -\alpha_{z} \epsilon_{z} \\
\eta_{x} \sigma_{\delta}^{2} & \eta_{x}^{\prime} \sigma_{\delta}^{2} & 0 & 0 & -\alpha_{z} \epsilon_{z} & \sigma_{\delta}^{2}
\end{array}\right),
$$

where $\sigma_{\delta}^{2}=\gamma_{z} \epsilon_{z}$. Moreover if we consider coupling in all three dimensions, the beam sigma matrix may be written as the usual symmetric variance matrix for a Gaussian distribution:

$$
\Sigma=\left(\begin{array}{cccccc}
\sigma_{x}^{2} & \sigma_{x x^{\prime}} & \sigma_{x y} & \sigma_{x y^{\prime}} & \sigma_{x z} & \sigma_{x \delta} \\
\sigma_{x x^{\prime}} & \sigma_{x^{\prime}}^{2} & \sigma_{x^{\prime} y} & \sigma_{x^{\prime} y^{\prime}} & \sigma_{x^{\prime} z} & \sigma_{x^{\prime} \delta} \\
\sigma_{x y} & \sigma_{x^{\prime} y} & \sigma_{y}^{2} & \sigma_{y y^{\prime}} & \sigma_{y z} & \sigma_{y \delta} \\
\sigma_{x y^{\prime}} & \sigma_{x^{\prime} y^{\prime}} & \sigma_{y y^{\prime}} & \sigma_{y^{\prime}}^{2} & \sigma_{y^{\prime} z} & \sigma_{y^{\prime} \delta} \\
\sigma_{x z} & \sigma_{x^{\prime} z} & \sigma_{y z} & \sigma_{y^{\prime} z} & \sigma_{z}^{2} & \sigma_{z \delta} \\
\sigma_{x \delta} & \sigma_{x^{\prime} \delta} & \sigma_{y \delta} & \sigma_{y^{\prime} \delta} & \sigma_{z \delta} & \sigma_{\delta}^{2}
\end{array}\right)
$$

which has 21 free parameters. The general Gaussian distribution is then given by

$$
f\left(x, x^{\prime}, y, y^{\prime}, z, \delta\right)=\frac{N \sqrt{|\Xi|}}{(2 \pi)^{3}} e^{-\frac{1}{2} \hat{\mathrm{X}}^{\mathrm{T}} \Xi \hat{\mathrm{X}}}
$$


where $\Xi=\Sigma^{-1}$.

The distribution given by Eq. 29 has a simple hyperelliptical shape. For a long beam passing through minimum of the beta-function, it should have a dog-bone shape. So far we have described the particle distribution relative to a longitudinal position $s$, where the $z$ coordinate is a time-like coordinate specifying how far the particle in question is in advance of the design particle. In order to evaluate the overlap integral of two colliding beams, we need to specify the density function in terms of $\left(x, x^{\prime}, y, y^{\prime}, s, \delta ; t\right)$, rather than $\left(x, x^{\prime}, y, y^{\prime}, z, \delta ; s\right)$. For a particle of velocity $v$, the relation between $s, z$, and $t$ is $z=v t-s$, and the required transformation between coordinates is defined by

$$
\hat{\mathrm{X}}\left(x, x^{\prime}, y, y^{\prime}, z, \delta ; t\right)=\mathrm{M}^{-1} \mathbf{X}\left(x, x^{\prime}, y, y^{\prime}, s, \delta ; t\right),
$$

i. e.,

$$
\left(\begin{array}{c}
x \\
x^{\prime} \\
y \\
y^{\prime} \\
z \\
\delta
\end{array}\right)=\left(\begin{array}{cccccc}
1 & -s & 0 & 0 & 0 & 0 \\
0 & 1 & 0 & 0 & 0 & 0 \\
0 & 0 & 1 & -s & 0 & 0 \\
0 & 0 & 0 & 1 & 0 & 0 \\
0 & 0 & 0 & 0 & 1 & -\frac{\beta s}{\gamma^{2}} \\
0 & 0 & 0 & 0 & 0 & 1
\end{array}\right)\left(\begin{array}{c}
x \\
x^{\prime} \\
y \\
y^{\prime} \\
v t-s \\
\delta
\end{array}\right)=\left(\begin{array}{c}
x-x^{\prime} s \\
x^{\prime} \\
y-y^{\prime} s \\
y^{\prime} \\
v t-\left(1+\frac{\beta \delta}{\gamma^{2}}\right) s \\
\delta
\end{array}\right)
$$

where $\beta=v / c$ and $\gamma=\sqrt{1-\beta^{2}}$ are the usual Lorentz parameters. Substituting Eq. 31 into Eq. 29 gives

$$
f\left(x, x^{\prime}, y, y^{\prime}, s, \delta ; t\right)=\frac{N \sqrt{|\Xi|}}{(2 \pi)^{3}} e^{-\frac{1}{2} \mathbf{X}^{\mathrm{T}}\left(\mathrm{M}^{-1}\right)^{\mathrm{T}} \Xi \mathrm{M}^{-1} \mathbf{X}},
$$

which will now have the correct dog-bone shape at the waist. For high energy bunched beams the $\frac{\beta \delta}{\gamma^{2}} \ll 1$ and may be ignored. (For RHIC $\frac{\beta \delta}{\gamma^{2}} \lesssim 10^{-7}$.) Integrating Eq. 32 over the momentum-like coordinates yields the particle density per volume as a function of spatial coordinate and time:

$$
\rho(x, y, s ; t)=\frac{N \sqrt{|\Xi|}}{(2 \pi)^{3}} \iiint e^{-\frac{1}{2} \mathbf{X}^{\mathrm{T}}\left(\mathrm{M}^{-1}\right)^{\mathrm{T}} \Xi \mathrm{M}^{-1} \mathbf{X}} d x^{\prime} d y^{\prime} d \delta .
$$

Let us now consider the limited case of a crossing point located in a straight section with no dispersion or horizontal-vertical coupling; here $\Xi$ of Eq. 25 becomes blockdiagonal:

$$
\Xi=\left(\begin{array}{cccccc}
\frac{\gamma_{x}}{\epsilon_{x}} & \frac{\alpha_{x}}{\epsilon_{x}} & 0 & 0 & 0 & 0 \\
\frac{\alpha_{x}}{\epsilon_{x}} & \frac{\beta_{x}}{\epsilon_{x}} & 0 & 0 & 0 & 0 \\
0 & 0 & \frac{\gamma_{y}}{\epsilon_{y}} & \frac{\alpha_{y}}{\epsilon_{y}} & 0 & 0 \\
0 & 0 & \frac{\alpha_{y}}{\epsilon_{y}} & \frac{\beta_{y}}{\epsilon_{y}} & 0 & 0 \\
0 & 0 & 0 & 0 & \frac{\gamma_{z}}{\epsilon_{z}} & \frac{\alpha_{z}}{\epsilon_{z}} \\
0 & 0 & 0 & 0 & \frac{\alpha_{z}}{\epsilon_{z}} & \frac{\beta_{z}}{\epsilon_{z}}
\end{array}\right)
$$




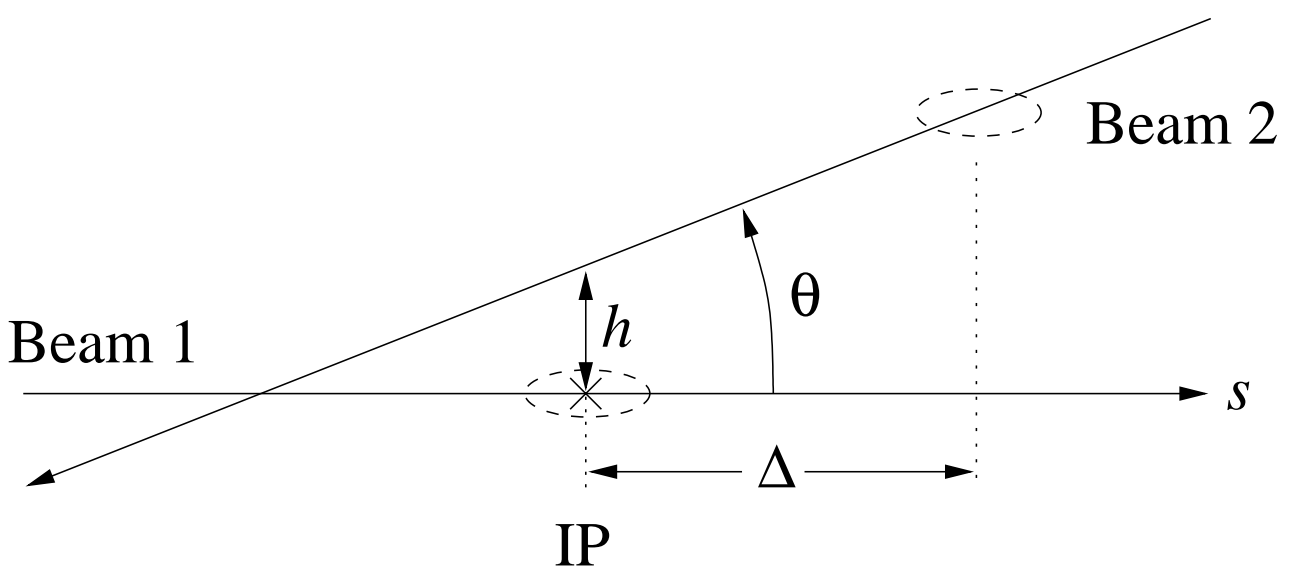

FiguRE 1. Geometry of errors at the beam's crossing point.

With this form, the integration of Eq. 33 is fairly simple and gives:

$$
\rho(x, y, s ; t)=\frac{N e^{-\frac{x^{2}}{2 \epsilon_{x}\left(\beta_{x}^{*}-2 \alpha_{x}^{*} s+\gamma_{x}^{*} s^{2}\right)}} e^{-\frac{y^{2}}{2 \epsilon_{y}\left(\beta_{y}^{*}-2 \alpha_{y}^{*} s+\gamma_{y}^{*} s^{2}\right)}} e^{-\frac{(v t-s)^{2}}{2 \epsilon_{z} \beta_{z}^{*}}}}{\sqrt{(2 \pi)^{3}\left(\beta_{x}^{*}-2 \alpha_{x}^{*} s+\gamma_{x}^{*} s^{2}\right)\left(\beta_{y}^{*}-2 \alpha_{y}^{*} s+\gamma_{y}^{*} s^{2}\right) \beta_{z}^{*} \epsilon_{x} \epsilon_{y} \epsilon_{z}}},
$$

where the superscript " $*$ "'s refer to the value at the design crossing point where $s=0$. It is worthwhile noting that the expressions of transverse Twiss parameters in parentheses are just the evolution of the transverse $\beta$-functions along $s$ :

$$
\beta_{x}(s)=\beta_{x}^{*}-2 \alpha_{x}^{*} s+\gamma_{x}^{*} s^{2}, \quad \text { and } \quad \beta_{y}(s)=\beta_{y}^{*}-2 \alpha_{y}^{*} s+\gamma_{y}^{*} s^{2} .
$$

For two colliding bunches the densities may be written as

$$
\rho_{1}(x, y, s ; t)=\frac{N_{1} e^{-\frac{x^{2}}{2 \epsilon_{x 1} \beta_{x 1}(s)}} e^{-\frac{y^{2}}{2 \epsilon_{y 1} \beta_{y 1}(s)}} e^{-\frac{(s-v t)^{2}}{2 \epsilon_{z 1} \beta_{z 1}^{*}}}}{\sqrt{(2 \pi)^{3} \epsilon_{x 1} \epsilon_{y 1} \epsilon_{z 1} \beta_{x 1}(s) \beta_{y 1}(s) \beta_{z 1}^{*}}}
$$

with the $s$-axis taken along the trajectory of beam-1 and

$$
\rho_{2}(x, y, s ; t)=\frac{N_{2} e^{-\frac{\left(x-h_{x}-\theta_{x s}\right)^{2}}{2 \epsilon_{x 2} \beta_{x 2}(s)}} e^{-\frac{\left(y-h_{y}-\theta_{y}\right)^{2}}{2 \epsilon_{y 2} \beta_{y 2}(s)}} e^{-\frac{(s+v t+\Delta)^{2}}{2 \epsilon_{z 2} \beta_{z 2}^{*}}}}{\sqrt{(2 \pi)^{3} \epsilon_{x 2} \epsilon_{y 2} \epsilon_{z 2} \beta_{x 2}(s) \beta_{y 2}(s) \beta_{z 2}^{*}}}
$$

the trajectory of beam-2 being offset at $s=0$ by $h_{x}$ in the $x$-direction and $h_{y}$ in the $y$-direction. (See Fig. 1.) Using a small angle approximation, the slopes of the second beam's trajectory in the $x$ and $y$ directions are respectively $\theta_{x}$ and $\theta_{y}$ relative to the first beam's trajectory. The parameter $\Delta$ accounts for a mistiming of the bunch crossing; the bunches then cross at $s=\Delta / 2$ from the design interaction point. The luminosity of this bunch crossing may be found by integrating the overlap of the two densities:

$$
L=2 v \iiint \int \rho_{1}(x, y, s ; t) \rho_{2}(x, y, s ; t) d x d y d s d t .
$$


Integrating the $x$-dependent exponentials over $x$ yields

$$
\int_{-\infty}^{\infty} e^{-\frac{\left(x-h_{x}-\theta_{x} s\right)^{2}}{2 \epsilon_{x 1} \beta_{x 1}(s)}} e^{-\frac{x^{2}}{2 \epsilon_{x 2} \beta_{x 2}(s)}} d x=\sqrt{\frac{2 \pi \epsilon_{x 1} \epsilon_{x 2} \beta_{x 1}(s) \beta_{x 2}(s)}{\epsilon_{x 1} \beta_{x 1}(s)+\epsilon_{x 2} \beta_{x 2}(s)}} e^{-\frac{\left(h_{x}+\theta_{x} s\right)^{2}}{2\left[\epsilon_{x 1} \beta_{x 1}(s)+\epsilon_{x 2} \beta_{x 2}(s)\right]}} .
$$

A similar integral occurs for the $y$-dimension. The integral of the $t$-dependent exponentials is

$$
\int_{-\infty}^{\infty} e^{-\frac{(s-v t)^{2}}{2 \epsilon_{z 1} \beta_{z 1}^{*}}} e^{-\frac{(s+v t+\Delta)^{2}}{2 \epsilon_{z 2} \beta_{z 2}^{*}}} v d t=\sqrt{\frac{2 \pi \epsilon_{z 1} \epsilon_{z 2} \beta_{z 1}^{*} \beta_{z 2}^{*}}{\epsilon_{z 1} \beta_{z 1}^{*}+\epsilon_{z 2} \beta_{z 2}^{*}}} e^{-\frac{(2 s+\Delta)^{2}}{2\left(\epsilon_{z 1} \beta_{z 1}^{*}+\epsilon_{z 2} \beta_{z 2}^{*}\right)}}
$$

Using these results Eq. 39 becomes

$$
L=\int_{-\infty}^{\infty} \frac{2 N_{1} N_{2} e^{-\frac{\left(h_{x}+\theta_{x s}\right)^{2}}{2\left[\epsilon_{x 1} \beta_{x 1}(s)+\epsilon_{x 2} \beta_{x 2}(s)\right]}} e^{-\frac{\left(h_{y}+\theta_{y} s\right)^{2}}{2\left[\epsilon_{y 1} \beta_{y 1}(s)+\epsilon_{y 2} \beta_{y 2}(s)\right]}} e^{-\frac{(2 s+\Delta)^{2}}{2\left(\epsilon_{z 1} \beta_{z 1}^{*}+\epsilon_{z 2} \beta_{z 2}^{*}\right)}}}{\sqrt{(2 \pi)^{3}\left[\epsilon_{x 1} \beta_{x 1}(s)+\epsilon_{x 2} \beta_{x 2}(s)\right]\left[\epsilon_{y 1} \beta_{y 1}(s)+\epsilon_{y 2} \beta_{y 2}(s)\right]\left[\epsilon_{z 1} \beta_{z 1}^{*}+\epsilon_{z 2} \beta_{z 2}^{*}\right]}} d s
$$

for a single crossing of two bunches.

For the special case where the colliding beams are collinear with identical beam sizes and shapes at the interaction point, and having $2 \sigma_{z} \lesssim \beta_{x}^{*}, 2 \sigma_{z} \lesssim \beta_{y}^{*}$, and $\Delta=0$, the previous integral simplifies to

$$
L=\frac{N_{1} N_{2}}{4 \pi \sigma_{x} \sigma_{y}} .
$$

The average instantaneous luminosity for $N_{b}$ crossing bunches per revolution with $N_{1}$ and $N_{2}$ particles per bunch respectively for the opposing beams is then

$$
\mathcal{L}=\frac{f_{0} N_{b} N_{1} N_{2}}{4 \pi \sigma_{x} \sigma_{y}}
$$

When the beams are round with $\epsilon_{\mathrm{rms}}=\epsilon_{x}^{\mathrm{rms}}=\epsilon_{y}^{\mathrm{rms}}$ and $\beta^{*}=\beta_{x}^{*}=\beta_{y}^{*}$ and of equal current, then

$$
\mathcal{L}=\frac{f_{0} N_{b} N^{2} \gamma}{4 \pi \beta^{*} \epsilon_{\mathrm{rms}}}
$$

In a more realistic situation the different bunches may have random intensities, so that the product $N_{b} N_{1} N_{2}$ must be replaced by a sum of the products of the colliding bunches' intensities, i. e.,

$$
N_{b} N_{1} N_{2} \rightarrow \Sigma_{j=1}^{N_{b}} N_{1 j} N_{2 j}
$$

\section{Estimation of LUminosities in RHIC}

Table I lists the RHIC design parameters for both protons and gold ions. The results of several simulated scans are shown in Figures 2 to 9 for both protons and gold ions. 
Table I: RHIC parameters

\begin{tabular}{|l|l|l|}
\hline Parameter & Protons & Gold Ions \\
\hline$\beta_{x \min }$ & $1.0 \mathrm{~m}$ & $1.0 \mathrm{~m}$ \\
$\beta_{y \min }$ & $1.0 \mathrm{~m}$ & $1.0 \mathrm{~m}$ \\
$\epsilon_{x 95 \%}^{N}$ & $20 \mu \mathrm{m}$ & $10 \mu \mathrm{m}$ \\
$\epsilon_{y 95 \%}^{N}$ & $20 \mu \mathrm{m}$ & $10 \mu \mathrm{m}$ \\
$\sigma_{z}$ & $0.075 \mathrm{~m}$ & $0.2 \mathrm{~m}$ \\
$N_{1}$ & $1 \times 10^{11}$ & $1 \times 10^{9}$ \\
$N_{2}$ & $1 \times 10^{11}$ & $1 \times 10^{9}$ \\
$N_{b}$ & 55 & 55 \\
$f_{\text {rev }}$ & $78.25 \mathrm{kHz}$ & $78.25 \mathrm{kHz}$ \\
$\gamma$ & 260 & 108 \\
\hline
\end{tabular}

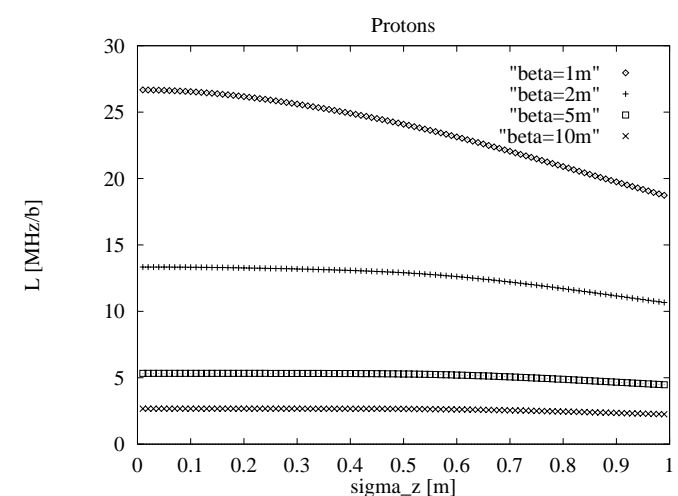

FiguRE 2. Luminosity versus bunch length for proton beams at various $\beta^{*}$ values.

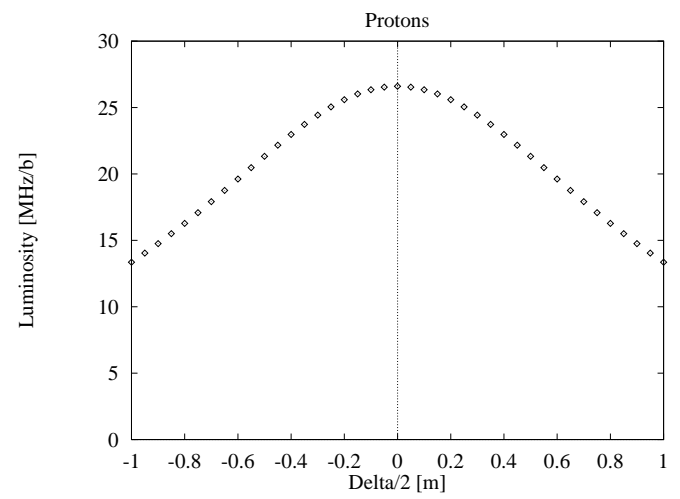

Figure 3. Longitudinal scan of luminosity for protons with $\beta^{*}=1 \mathrm{~m}$.

Fig. 2 shows the variation of the luminosity with bunch length for $\beta^{*}$ values from 1 to $10 \mathrm{~m}$. Here I have assumed that the beams are round and of the same size

$$
\beta_{x 1}^{*}=\beta_{y 1}^{*}=\beta_{x 2}^{*}=\beta_{y 2}^{*} \quad \text { and } \quad \epsilon_{x 1}^{*}=\epsilon_{y 1}^{*}=\epsilon_{x 2}^{*}=\epsilon_{y 2}^{*} .
$$

This demonstrates the usual rule of thumb that the bunch length should be no larger than the value of $\beta^{*}$

Fig. 5 shows the effect of misalignment of the waist of the the beam. Here I have assumed that the horizontal and vertical waists of both beams are displaced by the same amount but in opposite directions for both rings. This shows that to peak up luminosity, we should measure the optics carefully around the interaction regions. A scan of luminosity versus each waist should be made at some time to ensure that the optics are placing all four waists within about $10 \mathrm{~cm}$ of the interaction point.

The pair of beam position monitors (BPM) in each interaction region are separated by about $16.6 \mathrm{~m}$. The beam pipe aperture through the DX and D0 magnets limit the maximum crossing angle to about $1 \mathrm{mr}$. The alignment of the beams from the BPM's should be better than $200 \mu \mathrm{m}$, so that we should be able to dead reckon the crossing angle very well, $\theta_{x, y} \leq 6 \mu \mathrm{r}$. Figs. 6 and 7 show that there is very little degradation of 
luminosity for crossing angles up to several hundred microradians. Clearly the longer bunches of the gold beams are a bit more sensitive to crossing angle.

The rf system can easily phase the beams to cross at the required location within a couple of centimeters. Figs. 3 and 9 show that a $\Delta / 2=20 \mathrm{~cm}$ longitudinal shift of the IP has a marginal effect for both gold and proton beams

Since the BPM system should align the crossing point to a few hundred microns, we should be able to set the beam to collide with at least $30 \%$ of the peak luminosity. Even if we miss the crossing point by $0.5 \mathrm{~mm}$, we can perform a two dimensional scan in $100 \mu \mathrm{m}$ steps to get quite close to the peak. Then a finer transverse scan can be performed to reach the peak.

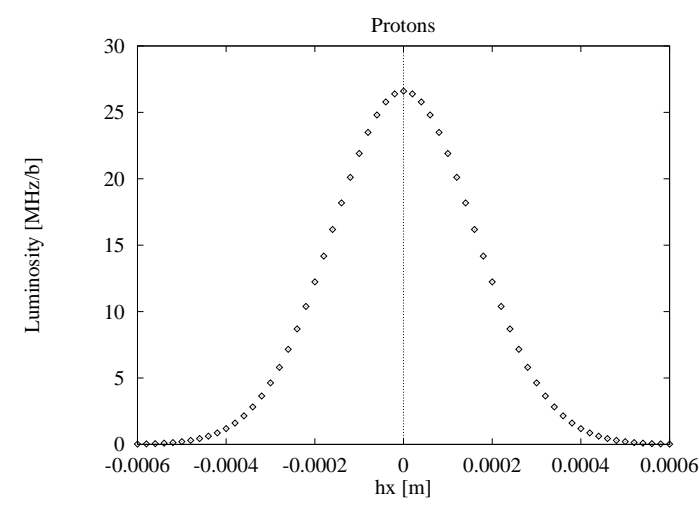

FiguRE 4. Scan of $h_{x}$ for proton beams at $250 \mathrm{GeV}$ with $\beta^{*}=1 \mathrm{~m}$. (The dot spacing is $20 \mu \mathrm{m}$.)

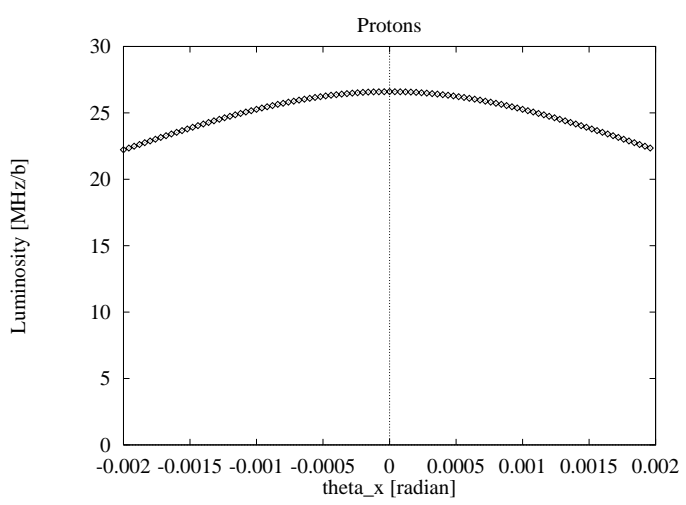

Figure 6. Scan of $\theta_{x}$ for proton beams at $250 \mathrm{GeV}$ with $\beta^{*}=1 \mathrm{~m}$.

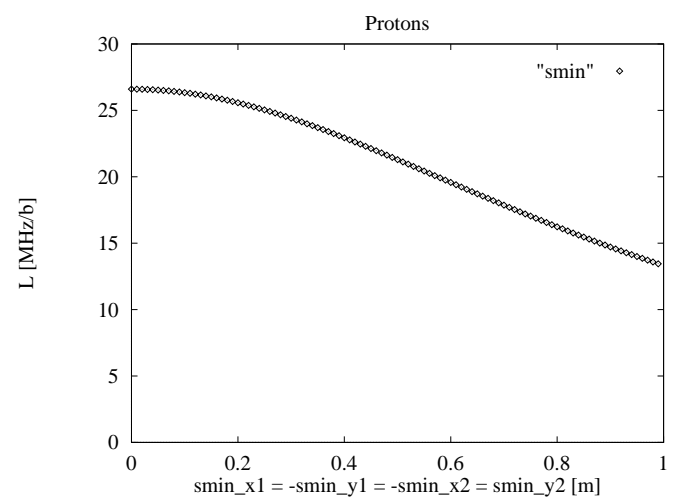

Figure 5. Longitudinal scan of beam waists for protons at $250 \mathrm{GeV}\left(\beta^{*}=\right.$ $1 \mathrm{~m})$.

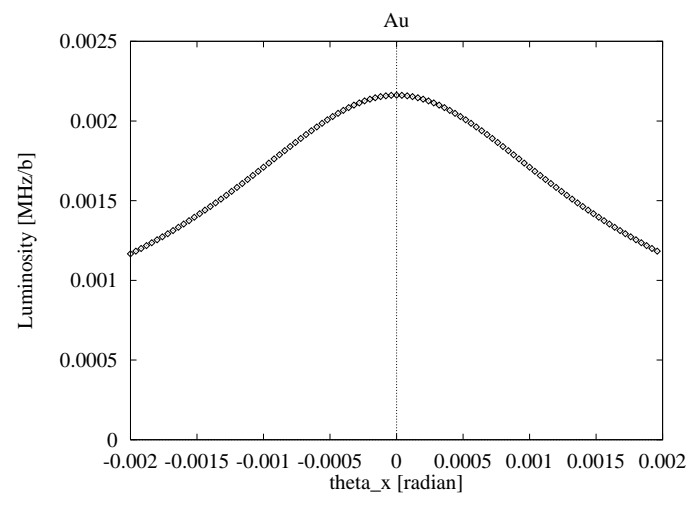

Figure 7. Scan of $\theta_{x}$ for Gold ion beams at $100 \mathrm{GeV} / \mathrm{amu}$ with $\beta^{*}=1 \mathrm{~m}$ and 55 bunches of $10^{9}$ ions per bunch. 


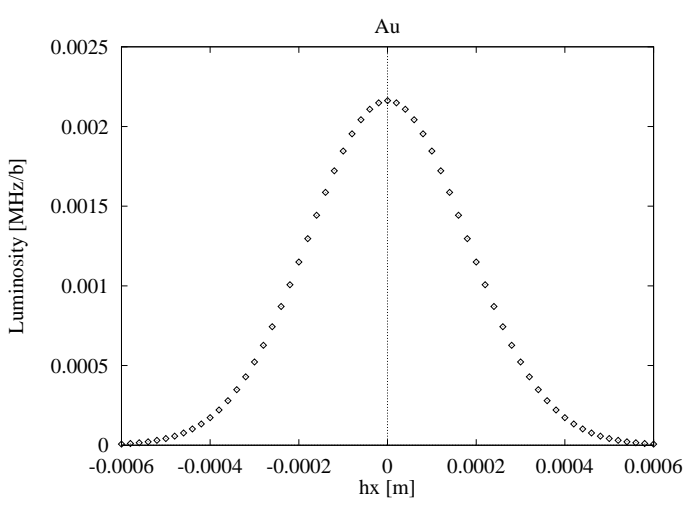

FiguRE 8. Scan of $h_{x}$ for Gold ion beams at $100 \mathrm{GeV} / \mathrm{amu}$ with $\beta^{*}=1 \mathrm{~m}$ and 55 bunches of $10^{9}$ ions per bunch. (The dot spacing is $20 \mu \mathrm{m}$.)

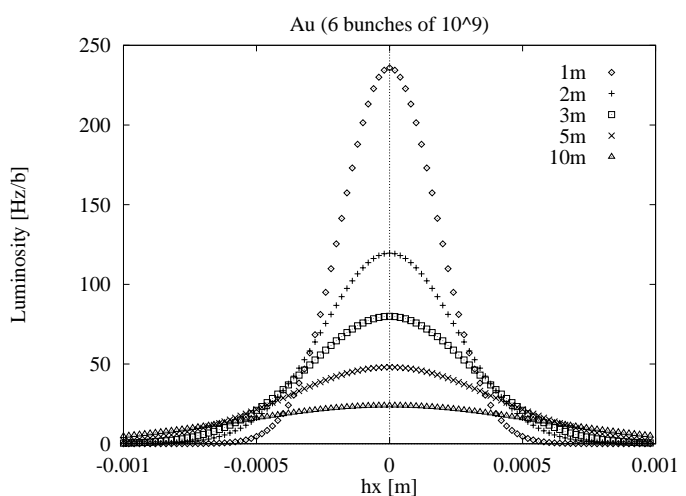

Figure 10. Scan of $h_{x}$ for Gold ion beams at $100 \mathrm{GeV} /$ amu with $\beta^{*}=1,2$, 3,5 , and $10 \mathrm{~m}$ and 6 bunches of $10^{9}$ ions per bunch. (The dot spacing is $20 \mu \mathrm{m}$.)

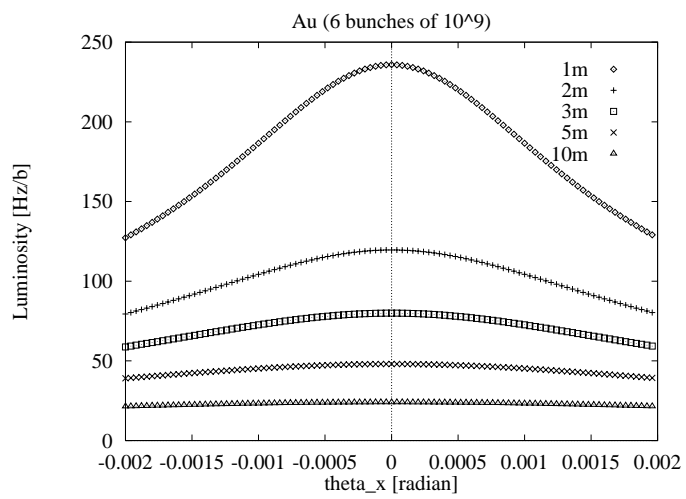

Figure 12. Scan of $\theta_{x}$ for Gold ion beams at $100 \mathrm{GeV} /$ amu with $\beta^{*}=1$, $2,3,5$, and $10 \mathrm{~m}$ and 6 bunches of $10^{9}$ ions per bunch.

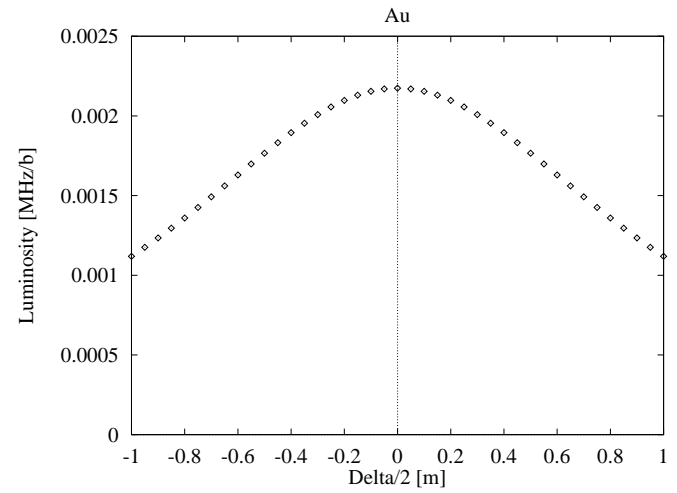

Figure 9. Longitudinal scan for Gold ion beams at $100 \mathrm{GeV} / \mathrm{amu}$ with $\beta^{*}=$ $1 \mathrm{~m}$ and 55 bunches of $10^{9}$ ions per bunch.

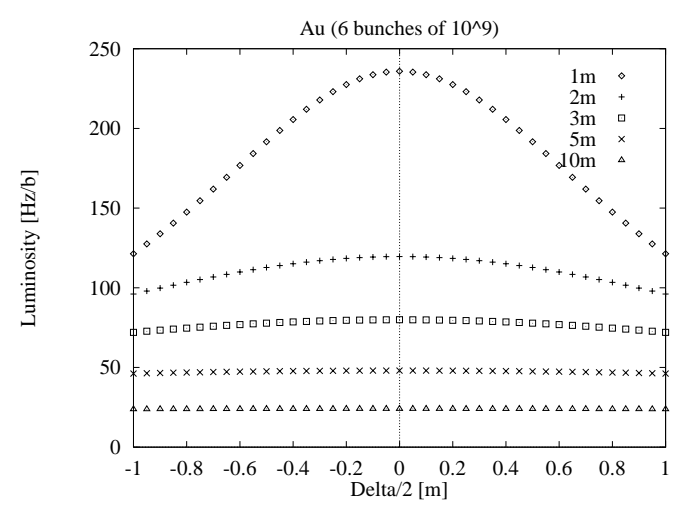

Figure 11. Longitudinal scan for Gold ion beams at $100 \mathrm{GeV} / \mathrm{amu}$ with $\beta^{*}=$ $1,2,3,5$, and $10 \mathrm{~m}$ and 6 bunches of $10^{9}$ ions per bunch. 